\title{
In Situ Continuous Wave Electron Paramagnetic Resonance Investigation of the Amyloid Aggregation of Parkinson's Protein Alpha-Synuclein-the Second Spin-Label Position
}

\author{
Enrico Zurlo ${ }^{1} \cdot$ Leonardo Passerini $^{1} \cdot$ Pravin Kumar $^{1} \cdot$ Martina Huber $^{1}$ D
}

Received: 5 June 2021 / Revised: 24 August 2021 / Accepted: 12 September 2021 /

Published online: 19 October 2021

(c) The Author(s) 2021

\begin{abstract}
Self-aggregation of amyloid proteins is a crucial step in neurodegenerative disease. The protein alpha-synuclein $(\alpha \mathrm{S})$ is implicated in Parkinson's disease. In an extension of the demonstration of in situ observation of intermediates in $\alpha \mathrm{S}$-aggregation by continuous wave (cw) EPR at room temperature (Zurlo et al. PLoS One 16: e0245548, 2021) by spin-label EPR, here the spin label is attached to position 90 $(\mathrm{R} 1 \alpha \mathrm{S} 90)$, rather than at position 56. The aim is to determine, if the spin-label position affects the kinetics of aggregation and if local information on the intermediates is accessible. Probed by the MTSL ((1-Oxyl-2,2,5,5-tetramethylpyrroline-3-methyl) methanethiosulfonate) spin label at position 90, using diamagnetic dilution of 9:1 wild type $\alpha \mathrm{S}$ to $\mathrm{R} 1 \alpha \mathrm{S} 90$, similar aggregation kinetics are found. Rotation correlation times for the spin label in the oligomer cannot be determined with sufficient accuracy to obtain local information on the oligomer under the conditions used. At the present stage, higher resolution EPR approaches, such as high-field EPR are more promising.
\end{abstract}

\section{Introduction}

Amyloid aggregation is a critical step in neurodegenerative disease and recent emphasis on oligomeric intermediates in this process as disease active agents has triggered the search for novel methods to measure such intermediates, see for example $[1,2]$. Alpha-synuclein $(\alpha \mathrm{S})$ is an interesting example, as it is a functional protein in the brain, where it is suggested to be involved in neuronal-vesicle fusion to the synapse, and thereby important in the neurotransmitter release in the synapse $[3,4]$. The protein $\alpha \mathrm{S}$ contains 140 aminoacids residues, lacks secondary structure

Martina Huber

huber@physics.leidenuniv.nl

1 Department of Physics, Huygens-Kamerlingh Onnes Laboratory, Leiden University, Niels Bohrweg 2, 2333 CA Leiden, The Netherlands 


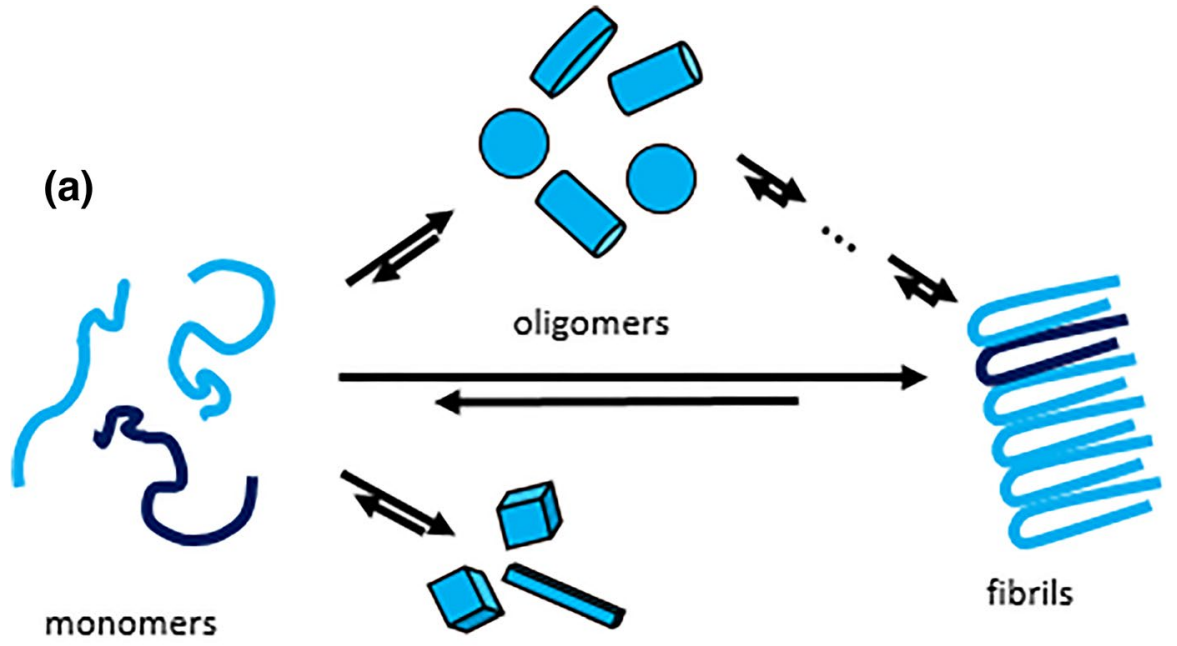

(b)

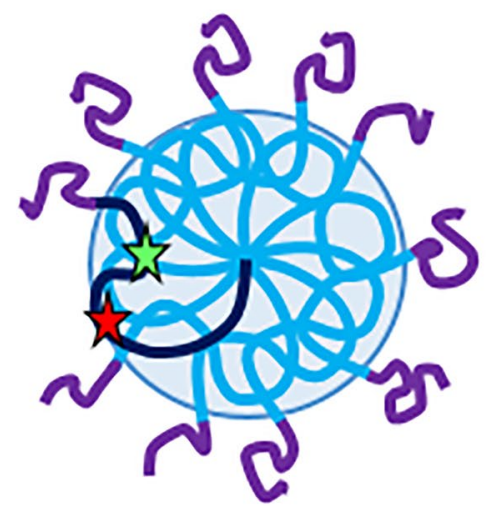

Fig. 1 Schematic overview of $\alpha \mathrm{S}$ aggregation processes and intermediates, from monomers to fibrils (shapes of oligomers are arbitrary and pathways serve as indications only) For kinetic interpretation, see Fig. 4 of [8] (a). b Schematic of hypothetical oligomer. Blue/black lines: $\alpha$ S, purple: C-terminal end, Red star: position 56, Green star: position 90 of $\alpha \mathrm{S}$. Lines without stars: wild type. For details, see text

in solution, and therefore is classified as an intrinsically disordered protein (IDP). It gained notoriety because of its putative role in Parkinson's disease [5, 6] and consists of the amphipatic region of residues 1-100, involved in membrane binding that also contains the NAC (non-amyloid-beta component) residues 61-95, and the negatively charged C-terminus, from residue 100 onwards [7].

In Fig. 1a, the overall process of amyloid self-aggregation, in which monomers aggregate into oligomers and fibrils is shown. Fibrils are elongated structures with a width of several $\mathrm{nm}$, in which the proteins adopt a characteristic cross- $\beta$-sheet secondary structure. Fibrils are the endpoint of aggregation, oligomers can be on or off pathway to fibrils, as shown in the top, respectively, bottom pathways in Fig. 1a. 
We have recently shown that by room temperature in situ $9 \mathrm{GHz}$ continuous wave (cw) EPR spectroscopy an oligomeric intermediate can be detected [8] and analysis with a kinetic model by Knowles et al. [9] was presented. It revealed a significant population of oligomers at early times of the aggregation, suggested that these oligomers are in equilibrium with monomers, and that monomers converted into fibrils [8].

In the present account, a second spin-label position is investigated and the EPR part of the study is elaborated further. An important observable of cw EPR is the rotation correlation time $\left(\tau_{\mathrm{r}}\right)$ of the nitroxide-part of the spin label. In nitroxide spin labels, the unpaired electron is delocalized on the nitrogen and oxygen atoms of the $\mathrm{N}-\mathrm{O}$-bond. As discussed earlier [8], $\tau_{\mathrm{r}}$ is related to the size of the particle the spin label is incorporated into, i.e. the size of the aggregate. For the MTSL spin-label ((1-Oxyl-2,2,5,5tetramethylpyrroline-3-methyl) methanethiosulfonate), additional degrees of freedom derive from rotation about the single bonds that link the nitroxide-ring to the protein backbone. The latter degree of freedom, referred to as the local mobility of the spin label, contains information about the immediate surrounding of the spin label: if the label is buried, its local mobility will be low, and its $\tau_{\mathrm{r}}$ will be longer than if the spin label is on the outside of an aggregate, where the $\tau_{\mathrm{r}}$ is expected to be smaller.

In the original study [8], $\alpha \mathrm{S}$ spin labeled at position 56 was investigated. For spin labeling, the $\alpha \mathrm{S}$ mutant with a cysteine at position 56 was labeled with the MTSL spin label. This construct is referred to in the following as R1 $\alpha$ S56. In the present study, the same approach is used, but now with the spin label attached to position 90, rather than 56 , R1 $\alpha$ S90. To investigate a second spin-label position serves two purposes: to investigate whether both constructs have similar aggregation kinetics and to see whether differences in the local mobility can be observed. The latter aspect would reveal structural features of the species investigated, as shown schematically in Fig. 1b, for a hypothetical oligomer. If the spin label is attached to a section of the protein that becomes buried in the aggregate (green star, Fig. 1b), it would have a lower mobility than a spin label attached to a position that remains on the outside of the respective aggregate (red star, Fig. 1b). As position 56 is in the center of the membrane-binding region and position 90 is towards the end of the NAC region, spin labels at these positions report on sections of the protein that likely differ in their involvement in oligomers and fibrils.

With respect to the first aim, we show that the label position does not affect the aggregation kinetics. A difference in local mobility at the two positions in the fibrilstate is likely, given what is presently known about $\alpha \mathrm{S}$ fibrils. We show that the experimental uncertainty in the present approach is too large to detect a possible difference in mobility in the fibrils. With respect to the oligomers, the superposition of monomer and oligomer spectra in the $9 \mathrm{GHz}$ EPR spectra creates a further obstacle. While $9 \mathrm{GHz}$ in situ cw EPR is valuable to track intermediates of aggregation and determine their kinetics, for information on their structures higher resolution, specialized EPR techniques are more promising. 


\section{Materials and Methods}

\subsection{Protein Preparation, Aggregation Conditions}

Protein expression and labeling were as described in Zurlo et al. [8]. Although the protein stocks were purified and monomeric, this was not tested immediately before starting the aggregation experiment, and therefore a small population of multimeric species cannot be excluded. Description of experiment: a stock solution of spinlabeled $\alpha$-synuclein (concentration between $150 \mu \mathrm{M}$ and $250 \mu \mathrm{M}$ ) was diluted into $3 \mathrm{~mL}$ of buffer (10 mM Tris-HCl, $\mathrm{pH} 7.4$ ), to a final concentration of $10 \mu \mathrm{M}$. The solution also contained $90 \mu \mathrm{M}$ wild-type $\alpha$-synuclein (wt- $\alpha \mathrm{S})$ for diamagnetic dilution (see below). The solution was divided into three $2 \mathrm{ml}$ LoBind Eppendorf tubes, resulting in a volume of $1.5 \mathrm{ml}$ per Eppendorf tube. The experiments were carried out over five days. After an initial measurement was taken at the time the spinlabeled protein was diluted $(t=0)$, the samples were allowed to aggregate on a thermomixer (Eppendorf, Thermomixer comfort) with a speed of $1000 \mathrm{rpm}$ at $37^{\circ} \mathrm{C}$. At each time point, $40 \mu \mathrm{L}$ samples were drawn from the aggregation solution and kept in the fridge at $4{ }^{\circ} \mathrm{C}$. From these, samples for EPR and Thioflavin T (ThT) fluorescence measurements were made. At the beginning, samples were collected every $3 \mathrm{~h}$, at later times the intervals were longer. These conditions correspond to those of the dataset HV1 and HV2 for R1 $\alpha$ S56 from Zurlo et al. [8]. For the EPR measurements, glass micropipettes of a volume of $50 \mu \mathrm{L}$ (Blaubrand Intramark, Wertheim, Germany) were filled with $20 \mu \mathrm{L}$ of the sample for each measurement.

\subsection{EPR Measurement Conditions}

The $9 \mathrm{GHz}$, continuous-wave EPR spectra were recorded using an ELEXSYS E680 spectrometer (Bruker, Rheinstetten, Germany). The measurements were done under the following conditions: room temperature, a microwave power of $0.63 \mathrm{~mW}$ and a modulation amplitude of $0.25 \mathrm{mT}$ at a modulation frequency of $100 \mathrm{kHz}$. The time expended on each measurement was adapted according to the spectral lineshape, i.e., the aggregation time, and they could last from 3 to $8 \mathrm{~h}$. Also for the R $1 \alpha \mathrm{S} 90$ sample, at long aggregation times the spectral amplitude decreases due to line broadening, and therefore, to obtain the desired signal-to-noise ratio, a longer accumulation time is needed. In practice, we inspected the signal-to-noise ratio of each EPR spectrum after a given accumulation time and increased the measurement time if the spectral quality was not yet sufficient [8]. The spin concentration was determined by comparing the double integral of the EPR spectra with the double integral of a reference sample (MTSL, $100 \mu \mathrm{M})$. The spin concentrations were $\approx 10 \mu \mathrm{M}$ for a total protein concentration of $100 \mu \mathrm{M}$. 


\subsection{Diamagnetic Dilution}

To suppress changes in the EPR lineshape by spin-spin interactions all samples were made with a mixture of 9:1 wild type aS: R1aS. This is expected to increase the spin-label to spin-label distance for the majority population of spin labels in aggregates to above 8-10 $\mathrm{A}$, which should be sufficient to suppress any visible effect of spin-spin interaction on the EPR lineshape. In the present experiment we want to avoid this interaction, as it would complicate/mask the line broadening by reduced mobility of the spin label upon aggregation.

\subsection{Simulations of EPR Spectra}

MATLAB (version 9.4.0.813654, R2018a, The MathWorks, Inc., Natick, MA, USA) and the EasySpin package (5.2.4) were used for simulations of the EPR spectra [10]. The simulations were performed as described by Zurlo et al. [8] which are reiterated for clarity here: the parameters of the simulation were manually adjusted to agree best with the experimental spectra. For all simulations, an isotropic rotation of the nitroxide $(S=1 / 2)$ and a common set of g-tensor principal values were used: $g=\left[\begin{array}{ll}2.009062 .006872 .00300\end{array}\right.$. A superposition of four components: two fast fractions using the "Garlic" function, and a medium and slow fraction using the "Chili" function. The principal values of the ${ }^{14} N$ hyperfine coupling tensor were $\mathrm{A}_{\mathrm{xx}}=\mathrm{A}_{\mathrm{yy}}=13 \mathrm{MHz}$ and $\mathrm{A}_{\mathrm{zz}}=110 \mathrm{MHz}$. For the slow component $\mathrm{A}_{\mathrm{zz}}=105 \mathrm{MHz}$ was used instead. A component line with a Gaussian shape and a width of $0.12 \mathrm{mT}$ was used for all simulations. The spectrum obtained at $t=0$ could be simulated with a single component, the fast fraction. The $\tau_{\mathrm{r}}$ obtained at $t=0$ was kept constant for all other simulations. From $t=3 \mathrm{~h}$ a new component appeared, $\tau_{\mathrm{r}}=0.24 \mathrm{~ns}$ which we attribute to free spin label. The subsequent time-point spectra also contained such a contribution, in the order of a few percent, but never exceeding $10 \%$. Optimal $\tau_{\mathrm{r}}$ values of the slow fraction were derived from the spectra at the end point of the aggregation. Similar to R $1 \alpha$ S56, the slow fraction was simulated with the "Chili" function. The $\tau_{\mathrm{r}}$ of $10 \mathrm{~ns}$ of the medium component was derived from spectra at times around $40-60 \mathrm{~h}$ of aggregation. The $\tau_{\mathrm{r}}$ values of each of the three fractions were kept constant for the entire series. For each time point, the relative contribution of the components was optimized.

Because of the diamagnetic dilution, which increases the spin-spin distance, the only EPR line broadening is expected to derive from mobility differences of the spin label: by diluting $\mathrm{R} 1 \alpha \mathrm{S}$ with wt- $\alpha \mathrm{S}$, the distances between the spin labels exceed the sensitive range of solution $\mathrm{cw}$ EPR, also in the aggregates.

\subsection{Analysis of EPR Parameters of R1aS90 With Respect to R1aS56}

Simulations were performed to check the sensitivity of the spectra to different $\tau_{\mathrm{r}}$ values of the fast, medium and slow components. In all the following tests, the contribution of each fraction to the respective spectrum was kept fixed. To test $\tau_{\mathrm{r}}$ 
of the slow fraction, spectra at late times of aggregation were simulated with different $\tau_{\mathrm{r}}$ times, revealing that changes in $\tau_{\mathrm{r}}$ of more than $0.01 \mathrm{~ns}$ for R $1 \alpha \mathrm{S} 56$ and more than $2.5 \mathrm{~ns}$ for $\mathrm{R} 1 \alpha \mathrm{S} 90$ resulted in visibly worse agreement of simulated and experimental spectra. The larger uncertainty margin for the R $1 \alpha \mathrm{S} 90$ mutant derives from a poorer signal-to-noise ratio compared to the R1 $\alpha \mathrm{S} 56$ spectra. For the fast component, spectra at the earliest aggregation times were used, revealing uncertainties in the range of $0.07 \mathrm{~ns}$. For the medium fraction, the range of acceptable $\tau_{\mathrm{r}}$ values is in the range of $3.5 \mathrm{~ns}$, significantly larger than for the fast and slow fractions. This is due to the lower amplitude that spectra with a longer $\tau_{\mathrm{r}}$ have and the superposition of the medium with the fast fraction, which both have intensity in the same spectral region. In spite of its even larger $\tau_{\mathrm{r}}$, the uncertainty of $\tau_{\mathrm{r}}$ of the slow fraction is smaller than that of the medium fraction, because the relative contribution of the slow fraction to the spectra is larger than that of the medium fraction, and, in addition, the slow fraction has intensity in magnetic-field regions where neither fast nor medium fraction contribute. The error margins are likely upper limits as only individual spectra are investigated, and not the entire series. The possibility of global fitting should be investigated in the future.

\section{Results}

Representative cw-EPR spectra of $\mathrm{R} 1 \alpha \mathrm{S} 90$, taken at different time points of the aggregation, are shown in Fig. 2, the full set in the appendix, Fig. 5. Three narrow lines, typical of the nitroxide in fast rotation, and, at later times, additional broad components are observed, the latter components are marked with an arrow in Fig. 2b-d.

Quantitative analysis of the spectra by simulations (see Materials and methods), performed by the same approach as [8], reveals the rotation correlation times of the three major fractions (see Table 1) and the amount by which each fraction contributes to the spectrum at the respective time point (see below).

Figure 3 shows the components of the simulation and how they sum up to the complete spectrum for one example, at $91 \mathrm{~h}$ of aggregation.

The second set of parameters that enters the analysis of the time course of the aggregation is the amount by which each of the component contributes to the spectra. It reflects the amount of the intermediates of aggregation. Figure 4 shows these amounts for a set of different aggregation experiments, in which Fig. 4a, b refer to the aggregation of $\mathrm{R} 1 \alpha \mathrm{S} 90$, and Fig. $4 \mathrm{c}$, $\mathrm{d}$ to $\mathrm{R} 1 \alpha \mathrm{S} 56$, taken from [8] as a reference.

Figure 4a shows the contributions for the dataset shown in Figs. 2 and $4 \mathrm{~b}$ the results of an independent aggregation experiment on a second sample of R $1 \alpha \mathrm{S} 90$. For the R1 $\alpha \mathrm{S} 90$ aggregation in Fig. $4 \mathrm{a}$ at early times, up to $10 \mathrm{~h}$, the fast fraction, which corresponds to monomers, dominates. In the time between 20 and $50 \mathrm{~h}$, the dominant aggregated species is a component with a $\tau_{\mathrm{r}}$ of $5 \mathrm{~ns}$, which, in accordance with Zurlo et al. [8] and as further discussed below (see Discussion), is attributed to an oligomeric state of $\alpha \mathrm{S}$. At late times, between 60 and $80 \mathrm{~h}$, the slow fraction, which is attributed to fibrils [8], raises. This fraction does not reach a plateau during 

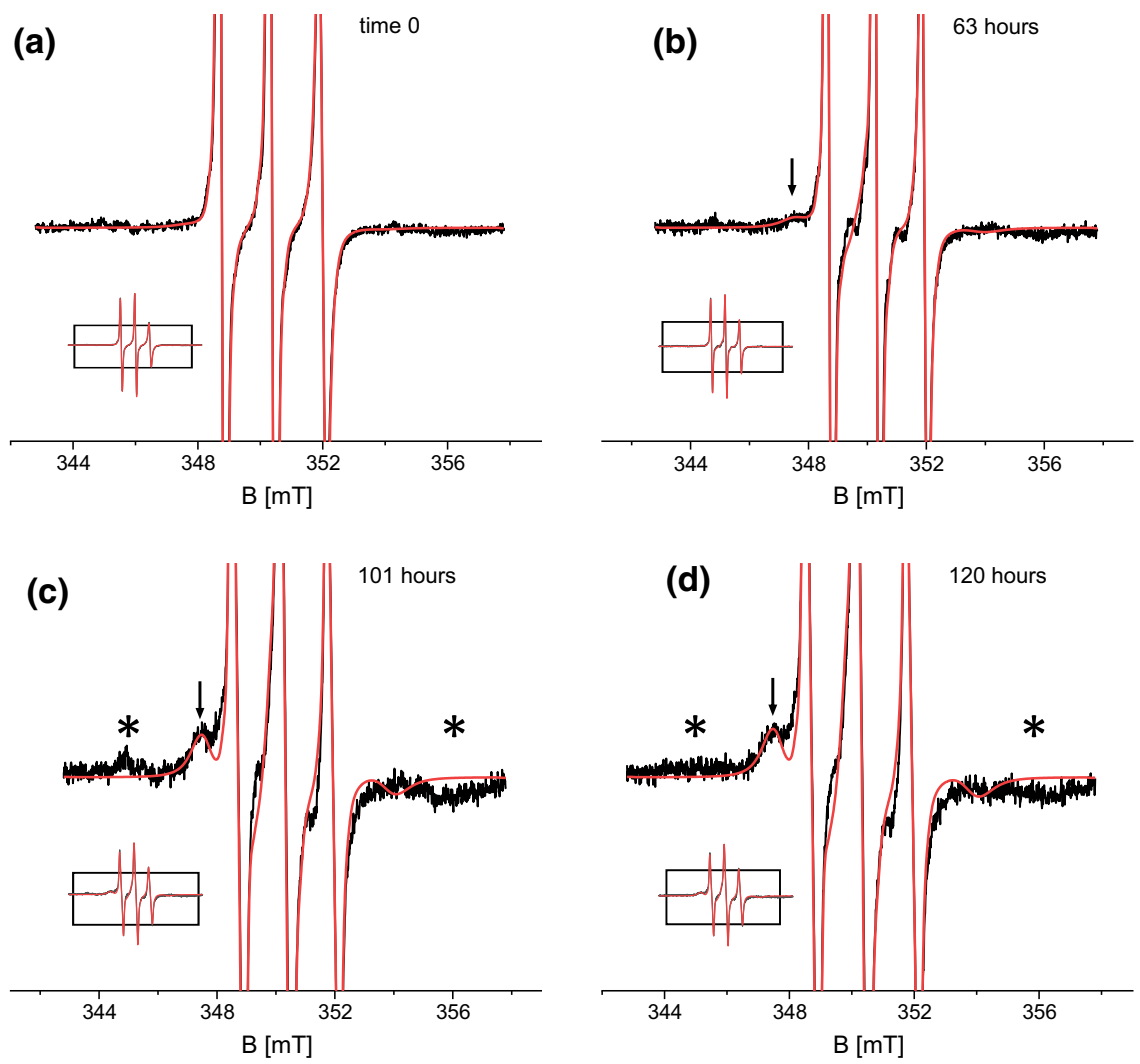

Fig. 2 Continuous wave EPR spectra of $\mathrm{R} 1 \alpha \mathrm{S} 90$ at selected time points of the aggregation. a Start of aggregation $(t=0)$. b $63 \mathrm{~h}$ of aggregation. c $101 \mathrm{~h}$ of aggregation. d $120 \mathrm{~h}$ of aggregation. Black: Experimental spectra. Red: Simulated spectra. Full spectrum: inset, box: area zoomed-in in enlarged spectrum. Amplitude enlarged by 2.5. Arrow: feature of broad spectral component. Asterisk: impurity signal

Table 1 Mobility of spin label in $\mathrm{R} 1 \alpha \mathrm{S}$ in the three fractions observed by EPR. Rotation correlation time: $\tau_{\mathrm{r}}$

\begin{tabular}{lllll}
\hline & Mutant & Fast & Medium & Slow \\
\hline$\tau_{\mathrm{r}}(\mathrm{ns})$ & $\mathrm{R} 1 \alpha \mathrm{S} 56$ & $0.43 \pm 0.06$ & $4.0 \pm 3.5$ & $10.00 \pm 0.01$ \\
$\tau_{\mathrm{r}}(\mathrm{ns})$ & $\mathrm{R} 1 \alpha \mathrm{S} 90$ & $0.37 \pm 0.07$ & $5.0 \pm 3.5$ & $10 \pm 2.5$ \\
\hline
\end{tabular}

the aggregation time monitored, because of the particular aggregation conditions. In ref. [8], two aggregation conditions are compared, the low volume (LV) and the high volume (HV) conditions. The results presented here for R1 $\alpha \mathrm{S} 90$ refer to the HV condition. Under HV conditions, the aggregation is slower than in LV condition, the slow fraction rises at a later time point, and does not reach a plateau, as demonstrated for $\mathrm{R} 1 \alpha \mathrm{S} 56$ in [8]. The same behavior is observed here for $\mathrm{R} 1 \alpha \mathrm{S} 90$. The independent aggregation experiment for $\mathrm{R} 1 \alpha \mathrm{S} 90$, Fig. $4 \mathrm{~b}$, reveals a similar pattern 


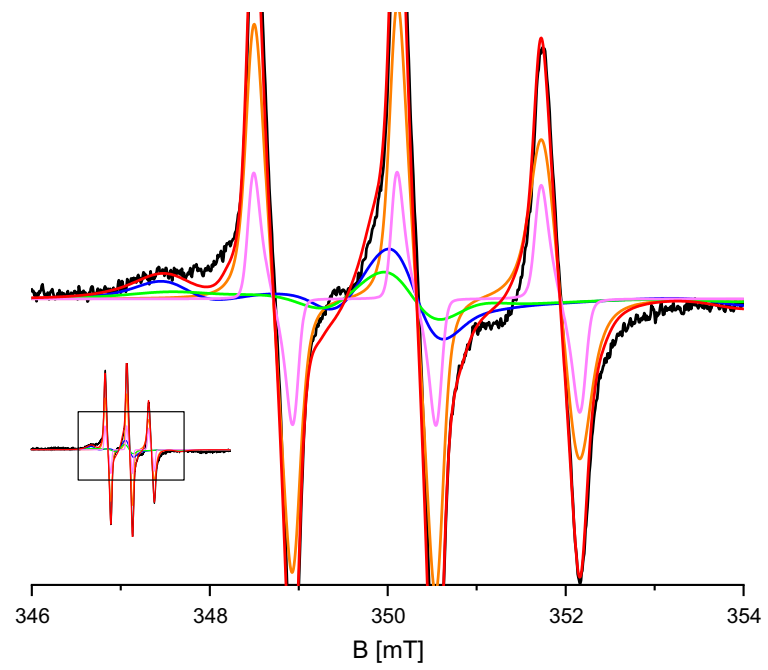

Fig. 3 Spectral components used in the simulation of the EPR spectra of $\alpha$-synuclein R1 $\alpha$ S 90 ( $91 \mathrm{~h}$ of aggregation). Full spectrum: inset, box: zoomed in area. Zoomed-in spectra: amplitude expanded by a factor 2.5 with respect to inset. Experimental spectrum (black), fast component (orange), medium component (blue), slow component (green), free spin label (pink). Total simulation (red). For details see text

to Fig. 4a, with small differences that reflect the sample to sample variations. Comparison with the equivalent data for $\mathrm{R} 1 \alpha \mathrm{S} 56$ (Fig. 4c, d), reveal an overall similar pattern to the data of R $1 \alpha \mathrm{S} 90$. Since the differences between the patterns of the two mutants are similar in magnitude to those between different samples of the same mutant, the aggregation kinetics must be similar. Therefore, the aggregation kinetics of R $1 \alpha \mathrm{S} 90$ follow those of R1 $1 \mathrm{~S} 56$, described in Zurlo et al. [8] for the HV conditions.

\section{Discussion}

In the present study we continue the investigation of the aggregation of $\alpha \mathrm{S}$ with the help of spin-label EPR. Attaching the spin label at position 90 (R1 1 S90) enables to study the aggregation at a protein position closer to the C-terminus than previously, where position 56 was targeted $(\mathrm{R} 1 \alpha \mathrm{S} 56)$ [8]. Here the EPR properties of the two positions are compared, with a special interest in the question if structural details can be derived from this kind of experiments.

For R $1 \alpha \mathrm{S} 90$, simulations required a minimum of three components (for details see Materials and methods). The $\tau_{\mathrm{r}}$ values of the fractions where determined from the spectra at carefully chosen aggregation times and the $\tau_{\mathrm{r}}$ values of monomers (fast fraction), and fibrils (slow fraction) were determined. The medium fraction is assigned to oligomeric intermediates. The reasoning is analogous to the one for the aggregation of R $1 \alpha S 56$ in [8]. Here the main lines are summarized: the smaller $\tau_{\mathrm{r}}$ value of the medium fraction compared to the fibril fraction, indicates 

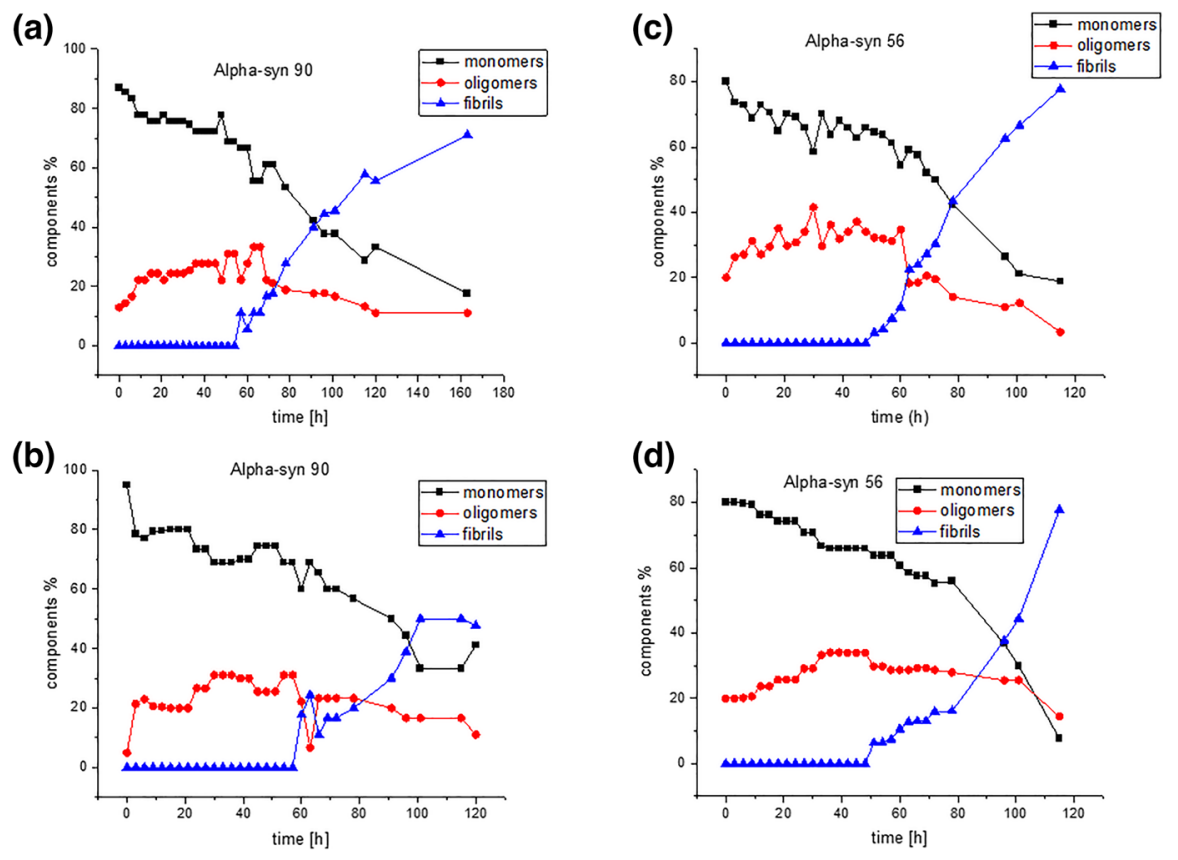

Fig. 4 Time course of aggregation for $\mathrm{R} 1 \alpha \mathrm{S} 90 \mathbf{a}, \mathbf{b}$ and comparison with $\mathrm{R} 1 \alpha \mathrm{S} 56 \mathbf{c}, \mathbf{d}$. Amounts by which three components contribute to the EPR spectra at different time points. Fast: monomer, medium: oligomer, slow: fibril (for details, see text)

smaller particles, and enhanced local mobility of the spin label in this fraction. The limitations of these conclusions are described in more detail in [8]. The maximum population of the medium fraction, in the order of $30 \%$, is observed before the slow fraction raises, an indication that the medium fraction is an intermediate of fibrillization, i.e. an oligomer. Given that the medium fraction appears before ThioT fluorescence sets in (see Fig. 6), and that ThioT fluorescence is an indicator for the formation of fibrils, the medium fraction does not contain fibrils. Considering the error margins of the $\tau_{\mathrm{r}}$ value of this fraction, see Materials and methods, the medium component is not necessarily a single species, suggesting that multiple oligomeric species could be formed during the time-frame when oligomers are observed.

The values of $\tau_{\mathrm{r}}$ and the amounts by which these fractions contribute to the spectra shows the similarity of the overall aggregation behavior for R $1 \alpha S 90$ and $\mathrm{R} 1 \alpha \mathrm{S} 56$. The similarity of the amount of each fraction as a function of time (Fig. 4) suggests that the kinetic analysis given in Zurlo et al. [8] is also valid here. Similar to the reporter position 56, the time dependence of the aggregation of $\mathrm{R} 1 \alpha \mathrm{S} 90$ shows the formation of an intermediate, assigned to an oligomeric state, with significant population, before fibrils are formed.

Similar aggregation kinetics of $\mathrm{R} 1 \alpha \mathrm{S} 56$ and R $1 \alpha \mathrm{S} 90$ is likely, because in both experiments a 9:1 excess of wild-type (wt) $\alpha \mathrm{S}$ relative to $\mathrm{R} 1 \alpha \mathrm{S}$ is used (diamagnetic dilution, see Materials and methods), and as a consequence, the aggregation 
should mostly be driven by the wt-protein, minimizing the influence of the spinlabel on the overall aggregation kinetics. In addition, compared to standard fluorescence labels, the spin label is relatively small and moderately polar, further reducing the possibility that the label affects the reaction. This justifies considering the spin label as a local reporter, rather than an agent in the reaction.

The dominant information contained in the $\tau_{\mathrm{r}}$ values, and therefore, the EPR spectra, is the local environment of the spin label and in particular the buriedness of the spin label. Figure 1b illustrates this in a cartoon form: different locations of the spin label, indicated as colored stars, will have different local environments. The section of $\alpha \mathrm{S}$ close to the green star is more buried in the aggregate, resulting in a longer $\tau_{\mathrm{r}}$ than for the spin label at the red star. A longer $\tau_{\mathrm{r}}$ thus reveals that the labeled protein section is in the interior of an aggregate, rather than on the outside. To illustrate this, some information about structural aspects that could affect mobility at position 90 and 56 differentially is collected. We start with the description of the fibrils, followed by the oligomeric intermediates known to date.

Tuttle and colleagues [11] identified a Greek key motif for the monomer in the fibril, in which the residue A90 is buried inside an hydrophobic pocket in the innermost portion of the Greek key, while in their structure residues 55-62 are disordered and solvent exposed. Those results agree with another NMR structure [12] in which A90 takes part in a beta strand and A56 is inside a beta turn, while the Meier group [13] solved the structure of a high pH fibril form, in which both A90 and A56 took part in the formation of beta strands motifs.

Guerrero-Ferreira et al. did extensive analysis of the fibril structure of a C-terminally truncated $\alpha \mathrm{S}$ with Cryo-EM $[14,15]$ They noticed differences from the structures solved with NMR and highlighted different polymorphs, with the final composition most likely depending on the experimental conditions. Nonetheless A90 is buried inside an hydrophobic region in all the polymorphs they identified, while A56 is solvent exposed in the proto-fibrils, with residues 52-57 forming the interface of two protofilaments inside a fibril. Another CryoEM structure solved on the full length protein has opposite chirality with respect to the above-mentioned [14, 15], but agrees with residues 50-57 taking part in the interaction between two protofilaments, and A90 buried in the fibril core [16]. On the other hand, another recent work identified two different fibril polymorphs, starting with the same protofilament kernel but different interfaces between the protofilaments: residues 47-56 or 68-78, with A56 being solvent exposed in the latter case [17]. Those experimental results agree with computational analysis that show how, in all fibril polymorphs, residue A90 takes part in a beta strand forming the hydrophobic core of the fibril $[18,19]$. So, in the selection of publications presented here, several structures suggest that position 90 would be more buried in the fibril than position 56, and, therefore, a spin label attached to position 90 may have a lower mobility than one at position 56.

With respect to $\alpha \mathrm{S}$ oligomers, structural information is much more scarce. Studies to track oligomers are mainly focused on shape and secondary structure determination, highlighting a highly heterogeneous composition, in which oligomers with varying degrees of $\beta$-sheet secondary structure, different shapes and properties were identified [20-25]. Some residue-level features were identified thanks to H/D exchange: In that case, the more protected regions were represented by the NAC core extending 
until A89, whereas the region extending between residues 55-76 appeared to be more dynamical [26]. Later, two different types of oligomers with different exchange patterns were identified, with A90 showing higher deuterium uptake with respect to A56 in both cases [27]. A site-specific fluorescence analysis [28] on residues 4,39,69,90,124 and 140 revealed that the residues up to and comprising A90, were forming the core of an oligomeric species, while the C-terminal sites remained solvent exposed. The buriedness, i.e. the low polarity of the fluorescent probe in position 90 in the oligomer was confirmed later in another study [29]. Overall, the scarceness of residue-specific structural information on oligomers in the literature illustrates how much need there is for methods that give structural information on these species. For oligomers, in addition, often specific conditions or chemical modifications are used to trap, stabilize or enrich an oligomeric species of interest. All these measures could have an influence on the oligomer structure. Consequently, in situ techniques that avoid isolation or enrichment techniques are particularly attractive.

The advantage of in situ EPR is that the aggregation is observed directly in solution. Therefore, there is no need for stabilization, isolation or manipulation of intermediates. Evidently, this includes intermediates that cannot be detected by traditional methods because, for example, they are not ThioT-active. The disadvantage is the superposition of different species that complicates the detection of individual spectra. In the case of $9 \mathrm{GHz} \mathrm{EPR}$, this particularly affects the intermediate state: the intermediate is a relatively broad component, the presence of which can be unambiguously determined, however, the lineshape of the spectra is less certain, as reflected in the large error margin of the $\tau_{\mathrm{r}}$ of the medium component (see Table 1). For the slow component, this disadvantage is compensated by its higher contribution in the late-time spectra, and the monomers can easily be detected, because, for $\alpha \mathrm{S}$, aggregation in quiescent solution at room temperature is sufficiently slow that the spectra of the monomers can be measured directly, i.e. without overlap of aggregated species. In addition, the small $\tau_{\mathrm{r}}$ of the monomers lead to narrow-line, high-amplitude EPR spectra that can easily be measured.

In the present study, we explore, if the approach presented in Zurlo et al. [8] can be expanded to obtain not only the kinetics of the development of species during amyloid aggregation, but also information on the structure of these species. Such information would be useful to understand the interconversion between oligomer and fibril, and also, in the case of harmful intermediates, aid to find inhibitors. It would provide complementary information to secondary structure content obtained by CD-spectroscopy [20,30], or shape, obtained by small angle X-ray scattering techniques [31]. We show that the spin label acts as a proper reporter and does not perturb the aggregation. However, under the conditions of the present experiments, the relatively large error margins of the experiment make it impossible to detect differences in local environment of the spin label at the positions 56 and 90 . Higher resolution techniques, such as high-field EPR [32] and specific methods of structure-analysis using distance determination by EPR will be better suited to reach that goal and are planned for the future. 


\section{Appendix}
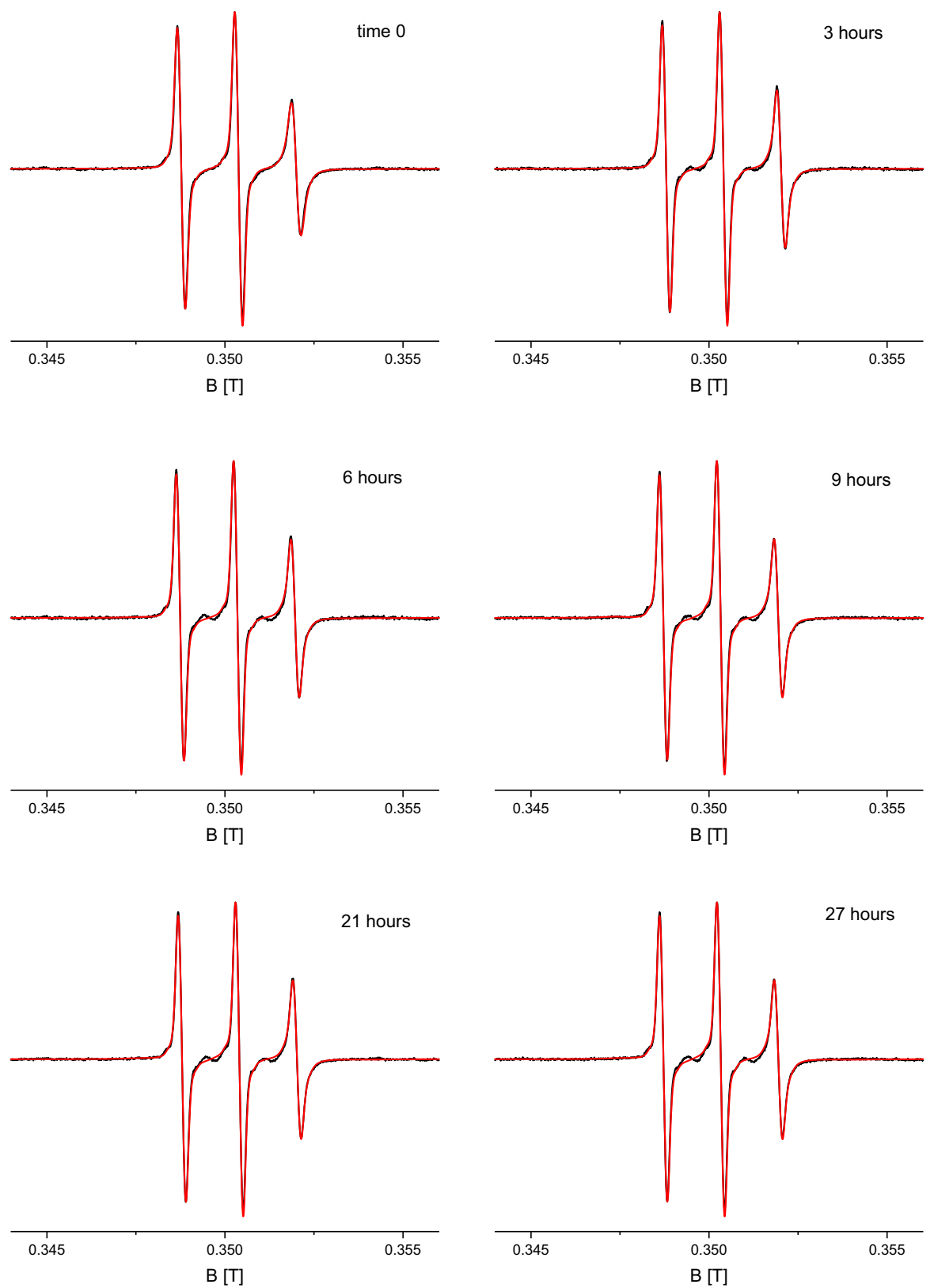

Fig. 5 EPR spectra of $\mathrm{R} 1 \alpha \mathrm{S} 90$ taken during the aggregation. Black: experimental, red: simulation 

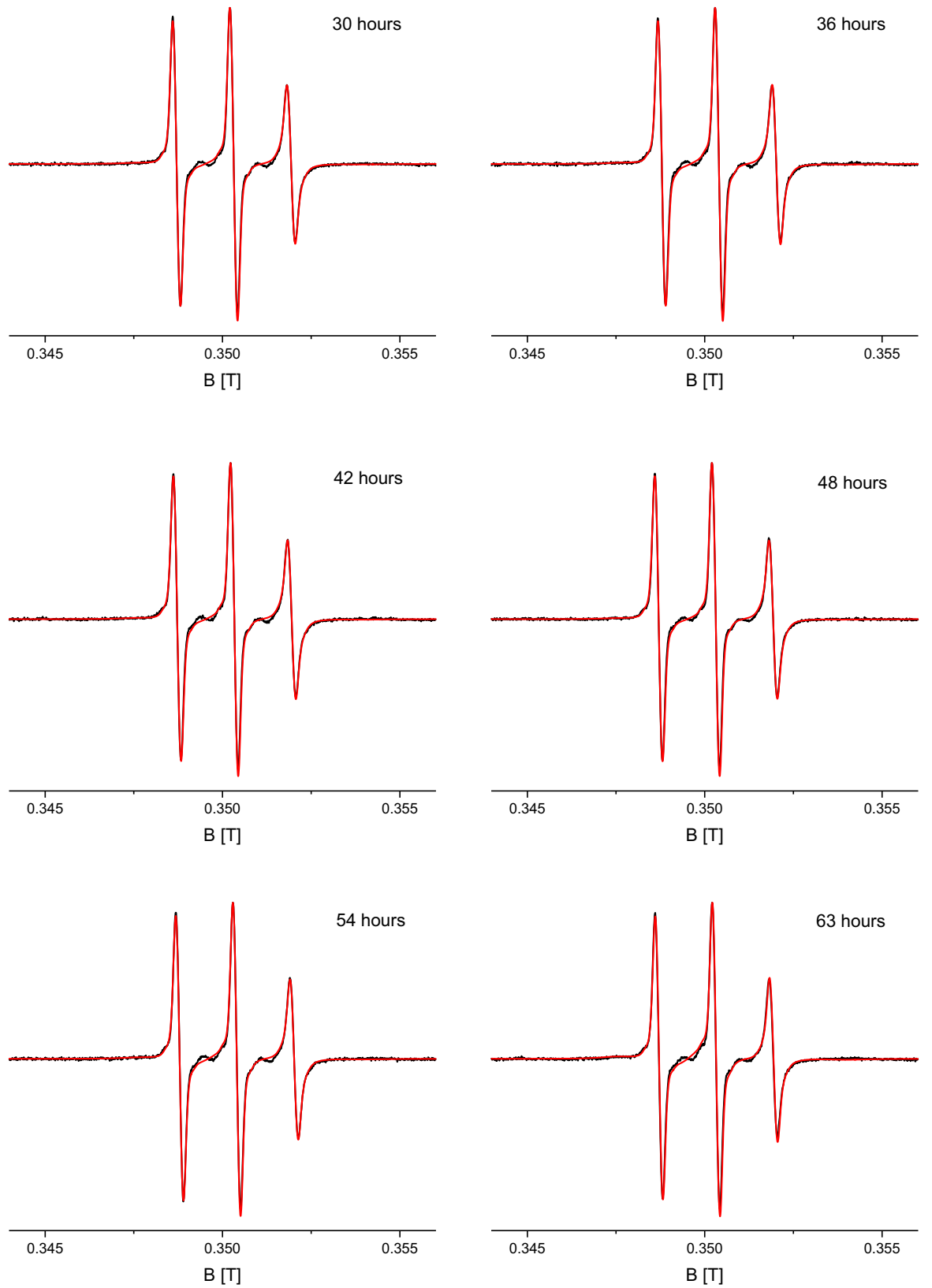

Fig. 5 (continued) 

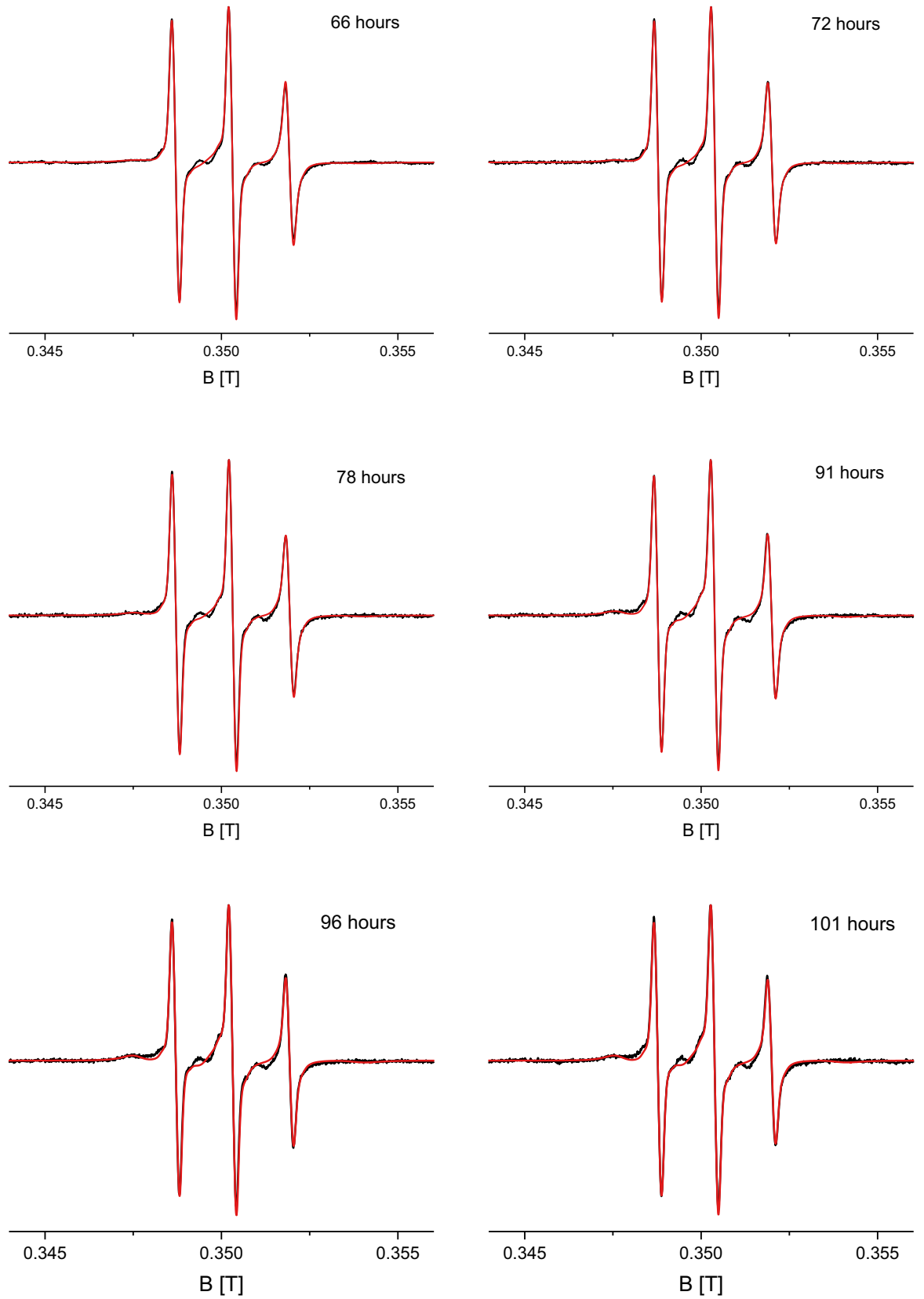

Fig. 5 (continued) 

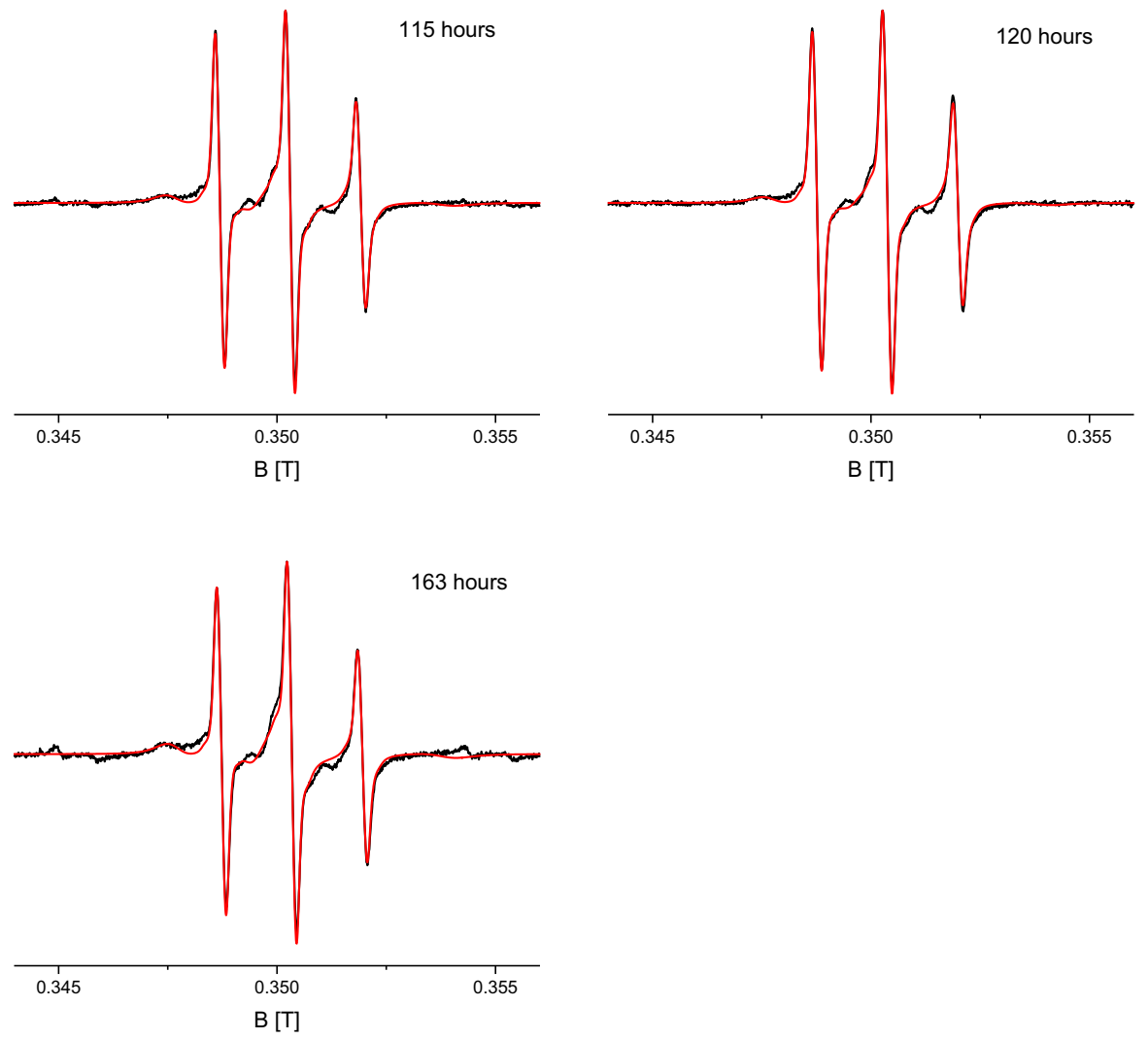

Fig. 5 (continued) 


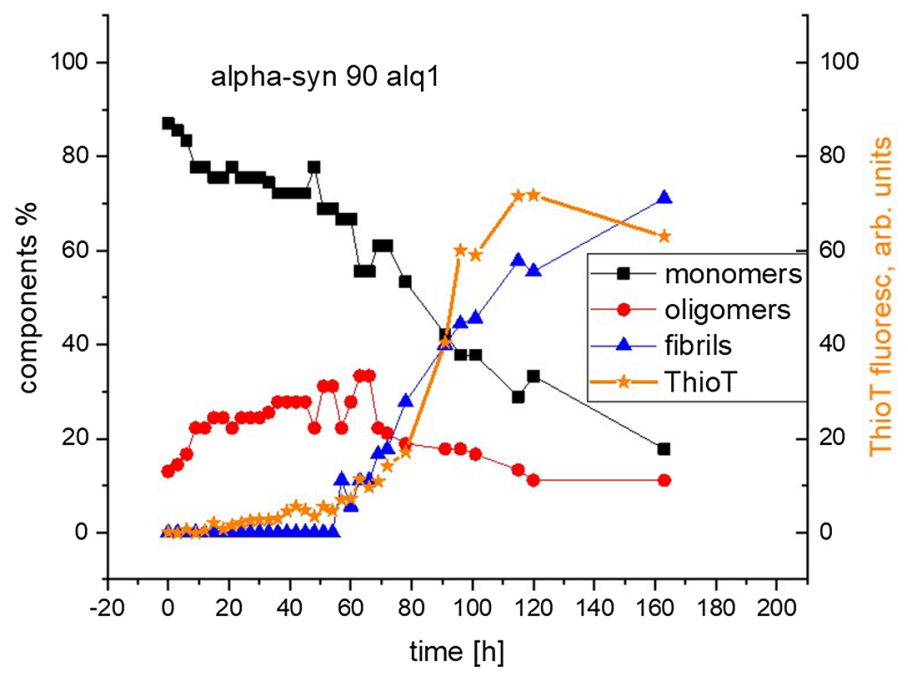

Fig. 6 Time course of aggregation for R $1 \alpha \mathrm{S} 90$ (see Fig. 4 b), including ThioT fluorescence data. Black, blue and red symbols: Amounts by which three EPR spectral components contribute to the spectra and ThioT fluorescence (orange stars). Fast: monomer, medium: oligomer, slow: fibril (for details, see text). ThioT fluorescence measurements: varian Cary Eclipse, San Jose, CA, USA. conditions: excitation wavelength: $457 \mathrm{~nm}$, fluorescence measured in the range of $475 \mathrm{~nm}$ to $600 \mathrm{~nm}$, fluorescence averaged between 485 and $490 \mathrm{~nm}$, i.e. the maximum of emission spectrum of ThioT, reference: ThioT in buffer

Acknowledgements We thank Mireille Claessens and her group, in particular Nathalie Schilderink, for the preparation of the spin-labeled and $w t-\alpha \mathrm{S}$ proteins. Financial support from the Dutch Organization for Scientific Research (NWO), grant 711.014.003 and grant 10SMPA04 in the context of the program entitled "A Single Molecule View on Protein Aggregation" from the Foundation for Fundamental Research on Matter (FOM), which is part of NWO, and The NanoFront program of Leiden and Delft Universities are gratefully acknowledged.

Open Access This article is licensed under a Creative Commons Attribution 4.0 International License, which permits use, sharing, adaptation, distribution and reproduction in any medium or format, as long as you give appropriate credit to the original author(s) and the source, provide a link to the Creative Commons licence, and indicate if changes were made. The images or other third party material in this article are included in the article's Creative Commons licence, unless indicated otherwise in a credit line to the material. If material is not included in the article's Creative Commons licence and your intended use is not permitted by statutory regulation or exceeds the permitted use, you will need to obtain permission directly from the copyright holder. To view a copy of this licence, visit http://creativecommons.org/licen ses/by/4.0/.

\section{References}

1. E.E. Cawood, T.K. Karamanos, A.J. Wilson, S.E. Radford, Biophys Chem 268, 106505 (2021). https://doi.org/10.1016/j.bpc.2020.106505

2. F. van Diggelen, A.W.J.W. Tepper, M.M. Apetri, D.E. Otzen, Isr J Chem 57, 699-723 (2017). https://doi.org/10.1002/ijch.201600116

3. D. Sulzer, R.H. Edwards, J Neurochem 150, 475-486 (2019). https://doi.org/10.1111/jnc.14810 
4. M.A.A. Fakhree, I.S. Nolten, C. Blum, M. Claessens, J Phys Chem Lett 9, 1249-1253 (2018). https://doi.org/10.1021/acs.jpclett.8b00092

5. T. Du, L. Wang, W. Liu, G. Zhu, Y. Chen, J. Zhang, Front Aging Neurosci 13, 645996 (2021). https://doi.org/10.3389/fnagi.2021.645996

6. S. He, S. Zhong, G. Liu, J. Yang, Neurodegener Dis 20, 55-64 (2020). https://doi.org/10.1159/ 000511083

7. D. Eliezer, E. Kutluay, R. Bussell Jr., G. Browne, J Mol Biol 307, 1061-1073 (2001). https://doi. org/10.1006/jmbi.2001.4538

8. E. Zurlo, P. Kumar, G. Meisl, A.J. Dear, D. Mondal, M. Claessens, T.P.J. Knowles, M. Huber, PLoS ONE 16, e0245548 (2021). https://doi.org/10.1371/journal.pone.0245548

9. R. Gaspar, G. Meisl, A.K. Buell, L. Young, C.F. Kaminski, T.P.J. Knowles, E. Sparr, S. Linse, Q Rev Biophys 50, e6 (2017). https://doi.org/10.1017/S0033583516000172

10. S. Stoll, A. Schweiger, J Magn Reson 178, 42-55 (2006). https://doi.org/10.1016/j.jmr.2005.08.013

11. M.D. Tuttle, G. Comellas, A.J. Nieuwkoop, D.J. Covell, D.A. Berthold, K.D. Kloepper, J.M. Courtney, J.K. Kim, A.M. Barclay, A. Kendall, W. Wan, G. Stubbs, C.D. Schwieters, V.M. Lee, J.M. George, C.M. Rienstra, Nat Struct Mol Biol 23, 409-415 (2016). https://doi.org/10.1038/nsmb.3194

12. J. Gath, L. Bousset, B. Habenstein, R. Melki, B.H. Meier, A. Bockmann, Biomol NMR Assign 8, 395-404 (2014). https://doi.org/10.1007/s12104-013-9526-y

13. J. Verasdonck, L. Bousset, J. Gath, R. Melki, A. Bockmann, B.H. Meier, Biomol NMR Assign 10, 5-12 (2016). https://doi.org/10.1007/s12104-015-9628-9

14. R. Guerrero-Ferreira, N.M. Taylor, A.A. Arteni, P. Kumari, D. Mona, P. Ringler, M. Britschgi, M.E. Lauer, A. Makky, J. Verasdonck, R. Riek, R. Melki, B.H. Meier, A. Bockmann, L. Bousset, H. Stahlberg, Elife (2019). https://doi.org/10.7554/eLife.36402.001

15. R. Guerrero-Ferreira, N.M. Taylor, D. Mona, P. Ringler, M.E. Lauer, R. Riek, M. Britschgi, H (2018) Elife. https://doi.org/10.7554/eLife.36402.001

16. Y. Li, C. Zhao, F. Luo, Z. Liu, X. Gui, Z. Luo, X. Zhang, D. Li, C. Liu, X. Li, Cell Res 28, 897-903 (2018). https://doi.org/10.1038/s41422-018-0075-x

17. B. Li, P. Ge, K.A. Murray, P. Sheth, M. Zhang, G. Nair, M.R. Sawaya, W.S. Shin, D.R. Boyer, S. Ye, D.S. Eisenberg, Z.H. Zhou, L. Jiang, Nat Commun 9, 3609 (2018). https://doi.org/10.1038/ s41467-018-05971-2

18. D.N. Bloch, Y. Miller, ACS Omega, 2 (2017) 3363-3370. https://scite.ai/reports/https://doi.org/ 10.1021/acsomega.7b00459

19. M. Pollock-Gagolashvili, Y. Miller, ACS Chem Neurosci 8, 2613-2617 (2017). https://doi.org/ 10.1021/acschemneuro.7b00334

20. S.W. Chen, S. Drakulic, E. Deas, M. Ouberai, F.A. Aprile, R. Arranz, S. Ness, C. Roodveldt, T. Guilliams, E.J. De-Genst, D. Klenerman, N.W. Wood, T.P. Knowles, C. Alfonso, G. Rivas, A.Y. Abramov, J.M. Valpuesta, C.M. Dobson, N. Cremades, Proc Natl Acad Sci U S A, 112 (2015) E1994-2003. https://scite.ai/reports/https://doi.org/10.1073/pnas.1421204112

21. N. Cremades, S.I. Cohen, E. Deas, A.Y. Abramov, A.Y. Chen, A. Orte, M. Sandal, R.W. Clarke, P. Dunne, F.A. Aprile, C.W. Bertoncini, N.W. Wood, T.P. Knowles, C.M. Dobson, D. Klenerman, Cell 149, 1048-1059 (2012). https://doi.org/10.1016/j.cell.2012.03.037

22. L. Zhou, D. Kurouski, Anal Chem 92, 6806-6810 (2020). https://doi.org/10.1021/acs.analchem.0c00593

23. N. Lorenzen, S.B. Nielsen, A.K. Buell, J.D. Kaspersen, P. Arosio, B.S. Vad, W. Paslawski, G. Christiansen, Z. Valnickova-Hansen, M. Andreasen, J.J. Enghild, J.S. Pedersen, C.M. Dobson, T.P. Knowles, D.E. Otzen, J Am Chem Soc 136, 3859-3868 (2014). https://doi.org/10.1021/ja411577t

24. F. van Diggelen, D. Hrle, M. Apetri, G. Christiansen, G. Rammes, A. Tepper, D.E. Otzen, PLoS ONE 14, e0213663 (2019). https://doi.org/10.1371/journal.pone.0213663

25. G. Meisl, J.B. Kirkegaard, P. Arosio, T.C. Michaels, M. Vendruscolo, C.M. Dobson, S. Linse, T.P. Knowles, Nat Protoc 11, 252-272 (2016). https://doi.org/10.1038/nprot.2016.010

26. S. Mysling, C. Betzer, P.H. Jensen, T.J. Jorgensen, Biochemistry, 52 (2013) 9097-9103. https://doi. org/10.1021/bi4009193

27. W. Paslawski, S. Mysling, K. Thomsen, T.J. Jorgensen, D.E. Otzen, Angew Chem Int Ed Engl 53, 7560-7563 (2014). https://doi.org/10.1002/anie.201400491

28. B.D. van Rooijen, K.A. van Leijenhorst-Groener, M.M. Claessens, V. Subramaniam, J Mol Biol 394, 826-833 (2009). https://doi.org/10.1016/j.jmb.2009.10.021

29. J.I. Gallea, M.S. Celej, J Biol Chem 289, 26733-26742 (2014). https://doi.org/10.1074/jbc.M114. 566695 
30. T. Kamiyoshihara, M. Kojima, K. Ueda, M. Tashiro, S. Shimotakahara, Observation of multiple intermediates in alpha-synuclein fibril formation by singular value decomposition analysis. Biochem Biophys Res Commun 355, 398-403 (2007). https://doi.org/10.1016/j.bbrc.2007.01.162

31. L. Giehm, D.I. Svergun, D.E. Otzen, B. Vestergaard, Low-resolution structure of a vesicle disrupting alpha;-synuclein oligomer that accumulates during fibrillation, Proc Natl Acad Sci U S A, 108 (2011) 3246-3251.https://doi.org/10.1073/pnas.1013225108

32. K. Möbius, W. Lubitz, A. Savitsky, High-field EPR on membrane proteins - crossing the gap to NMR. Prog. Nucl. Magn. Reson. Spectrosc. 75, 1-49 (2013). https://doi.org/10.1016/j.pnmrs.2013. 07.002

Publisher's Note Springer Nature remains neutral with regard to jurisdictional claims in published maps and institutional affiliations. 